\title{
BRAIN STEM MECHANISMS CONTROLLING THE JAW MUSCLE-TONUS OF THE DOG*
}

\author{
YOJIRO KAWAMURA, MASAYA FUNAKOSHI, SHUSAKU TSUKAMOTO \\ AND MITSURU TAKATA** \\ Department of Oral-Physiology, Dental, School, Osaka University Kitaku, Osaka, Japan
}

In the previous paper (1) we clearly demonstrated the distribution of the points in the midbrain and medulla of dogs which responded to depressing of the lower jaw, and the electrophysiological characteristics of this response were also analyzed. That is, regular spontaneous unit discharges (the background activities) which were recorded from and along the mesencephalic trigeminal nucleus and root were remarkably inhibited by elevating the lower jaw and accelerated by depressing the jaw. These responses were obtained by strictly localized punctures in the brainstem, accompanied by a short latency and slow adaptation as shown in goat by Cooper, Daniel and Whitterige (2).

These responses were also recorded at points in the trigeminal motor nucleus and lateral reticular formation at the level of the brachium pontis. Conversely, the spontaneous electrical activities of some of the points in the medulla near the hypoglossal root and nucleus were inhibited by depressing the lower jaw.

In the present paper the functional correlationships between these mesencephalic, pontine and bulbar points were analyzed, and additionally the principal factor inducing this response was determined.

\section{METHOD}

Ten adult dogs, all of them precollicular decerebrate and decerebellate animals, were used. The experimental procedures, the method of fixing the animal's head and to record the brain activity were similar as to those reported in the previous report. But in this experiment, two recording needle microelectrodes $20 \mu$ at the tip (each of them enclosed in a fine glass capillary) were simultaneously inserted into the brainstem stereotaxically. By these two electrodes activated responses of the mesencephalic trigeminal nucleus or bulbar trigeminal motor nucleus and the inhibited responses in the lower medulla were simultaneously recorded.

In addition, in some experiments, to solve the functional correlations existing between these central structures and each masticatory muscle, i.e. masseter,

\footnotetext{
Received for publication July 1, 1959.

* Presented in part at the 35th Annual Meeting of the Physiological Society of Japan, Kanazawa, May 4, 1958.

** 河村洋二郎, 船越正也, 塚本周作, 高田 充
} 
temporalis and occipito-mandibularis (digastric muscle of human-being), the muscles were separately stretched and the responses from the upper described central areas recorded. In this experiment, each masticatory muscle was previously separated from the mandibular bone so as to allow each to be stretched individually; a small fishhook connected to the load (100 g) by a cotton thread was attached at the free end of the muscle and the muscle was stretched mechanically by hanging the load.

The site of the recording electrode was verified histologically.

RESULTS

\section{Functional correlationships of the responded points in the brainstem}

The background activities of the mesencephalic nucleus and motor nucleus of the trigeminal nerve were accelerated simultaneously with a short latency in almost a similar pattern by depression of the lower jaw. Fig. 1 shows the simultaneous recording from the two points described above, the spontaneous discharges of both points were accelerated without a remarkable difference in delay during depression of the jaw. Adaptation of both of the responses

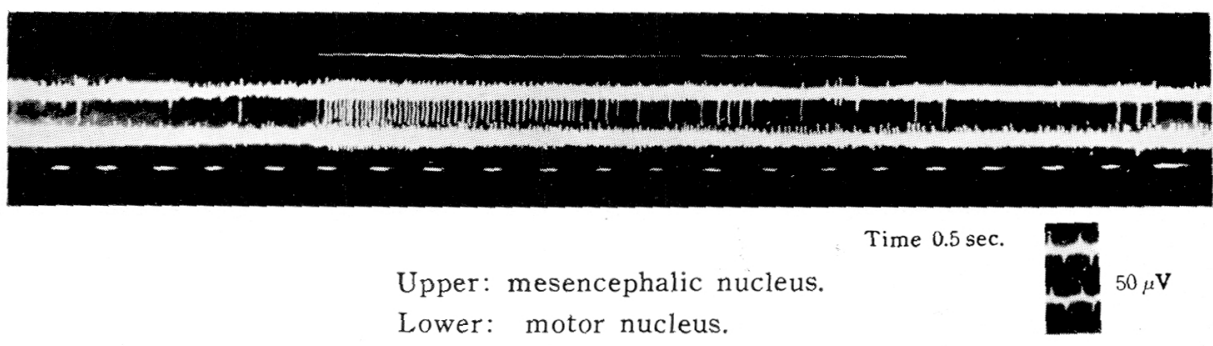

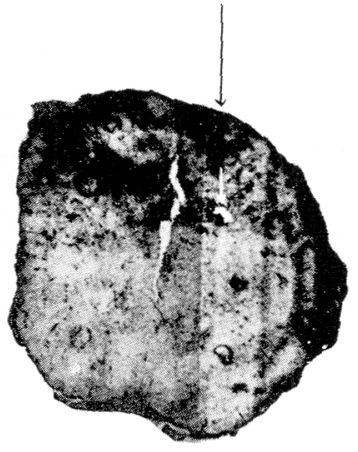

Mesencephalic nucleus of the fifth cranial nerve

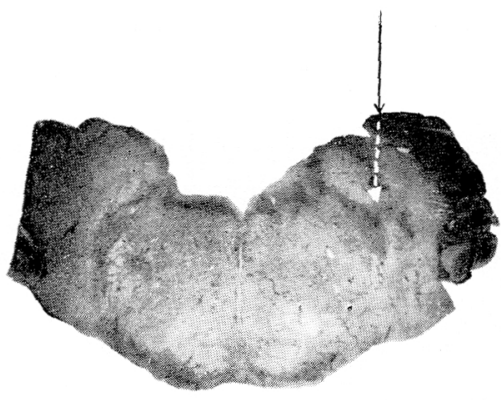

Motor nucleus of the fifth nerve

Site of electrode tip

FIG. 1. Simultaneous recording of activating response in mesencephalic nucleus and motor nucleus of trigeminal nerve. 
was slow, however, appearing somewhat earlier in the mesencephalic nucleus than in the motor nucleus of the trigeminal nerve. That is, in one case, about $1 \mathrm{c} / \mathrm{s}$ of the background activity of the mesencephalic nucleus was activated to about $20 \mathrm{c} / \mathrm{s}$ directly after loading, decreased to about $16 \mathrm{c} / \mathrm{s}$ after $2 \mathrm{sec}$. and reached about $3 \mathrm{c} / \mathrm{s}$ after $4 \mathrm{sec}$. But about $32 \mathrm{c} / \mathrm{s}$ of the background activity of the motor nucleus was constantly activated to about $71 \mathrm{c} / \mathrm{s}$ directly after loading, decreased to about $50 \mathrm{c} / \mathrm{s}$ after $2 \mathrm{sec}$. and reached about $37 \mathrm{c} / \mathrm{s}$ after $4 \mathrm{sec}$. This relation is shown in fig. 2 by a histogram of the unit discharge per second of these two points. Additionally, a suppression of discharge after releasing the tension was more dominant and long lasting in the mesencephalic response than in that of the motor nucleus of the trigeminal nerve.

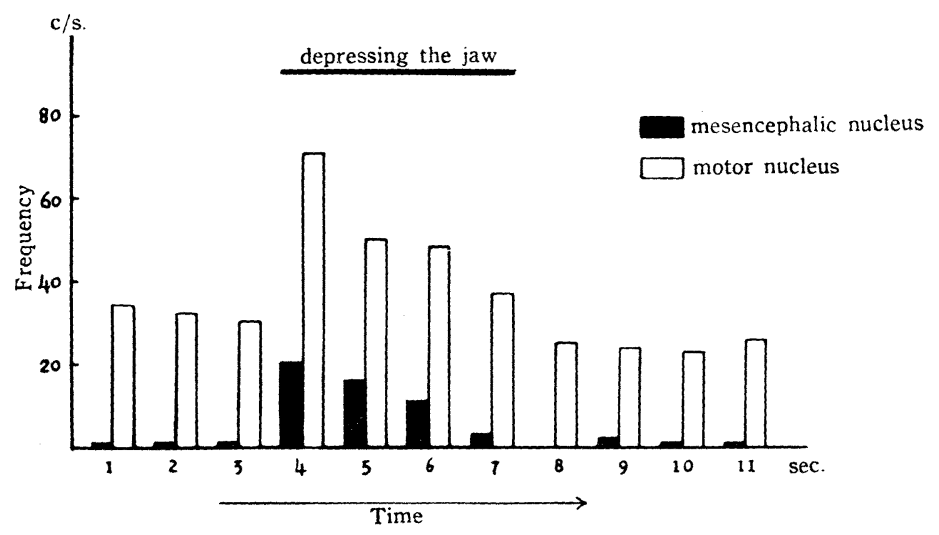

FIG. 2. Histogram of a discharge frequency in process of time in the mesencephalic and motor nucleus of the trigeminal nerve.

The spontaneous unit discharge of the mesencephalic and bulbar points was accelerated by depressing the lower jaw by the same method mentioned above, but conversely that of the hypoglossal nucleus was inhibitcd at the same time. Fig. 3 is one sample of a simultaneous recording of responses of the mesencephalic trigeminal nucleus and hypoglossal nucleus. Fig. 4 shows a histogram of the discharge pattern of these two points.

When the jaw closing muscles were relaxed by passive closure of the lower jaw, as shown in fig. 5 , the spontaneous discharge of the mesencephalic trigeminal nucleus was diminished reflexly but the discharge pattern of the hypoglossal nucleus was not affected noticeably.

2. Affection of a spindle afferent from each masticatory muscle on the trigeminal motor nucleus

In this experiment a recording microelectrode was inserted into the trigeminal motor nucleus which responded to depression of the lower jaw. Thenceforth, a response at this point was recorded during stretching by each masticatory muscle; the masseter, temporalis and occipito-mandibular muscle were stretched one by one successively. As shown in fig. 6, as an example, 


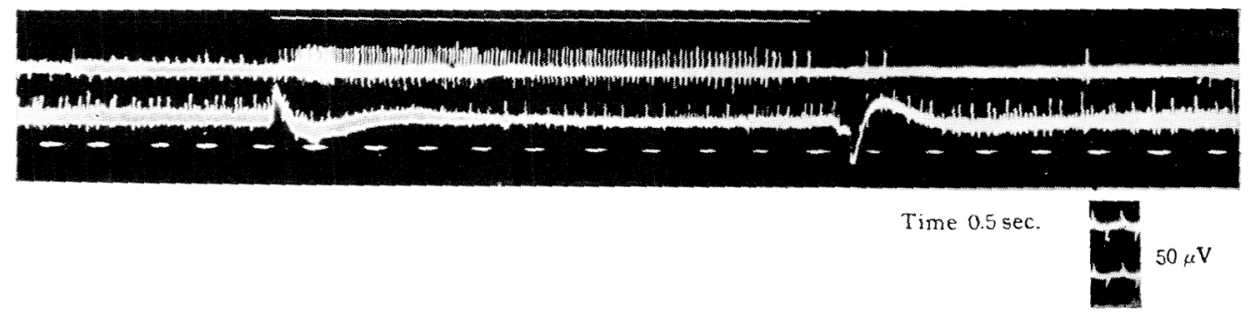

Upper: mesencephalic nucleus.

Lower: hypoglossal nucleus.

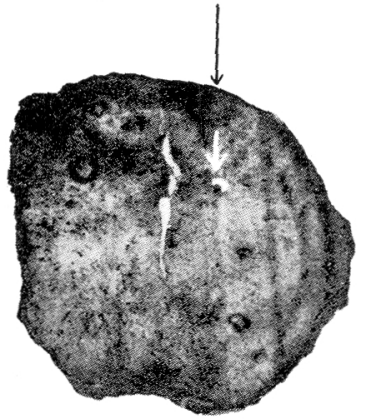

Mesencephalic nucleus of

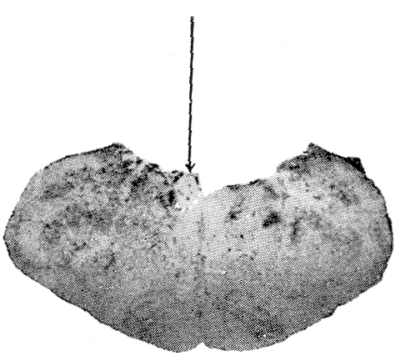

Hypoglossal nucleus the fifth cranial nerve

Site of electrode tip

FIG. 3. Simultaneous recording of inhibitory response in medulla and activating response in midbrain by depressing the jaw.

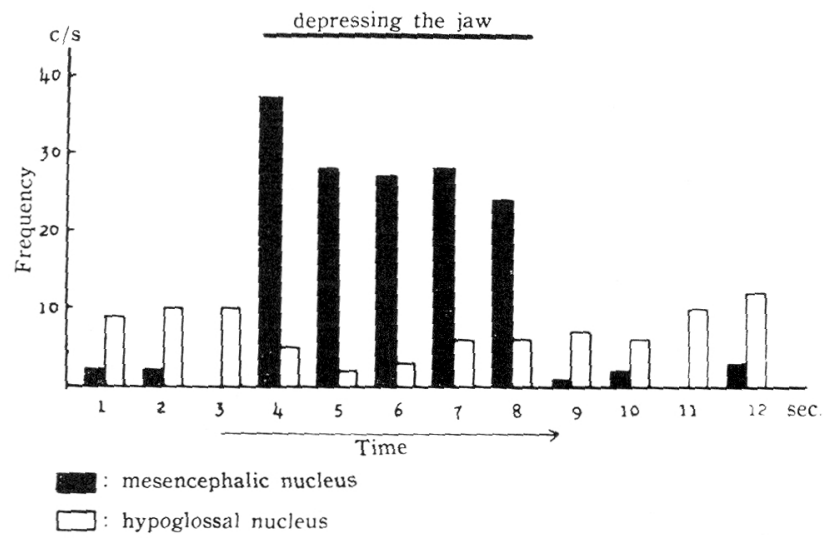

FIG. 4. Histogram of a discharge frequency in process of time in the mesencephalic trigeminal nucleus and hypoglossal nucleus. 


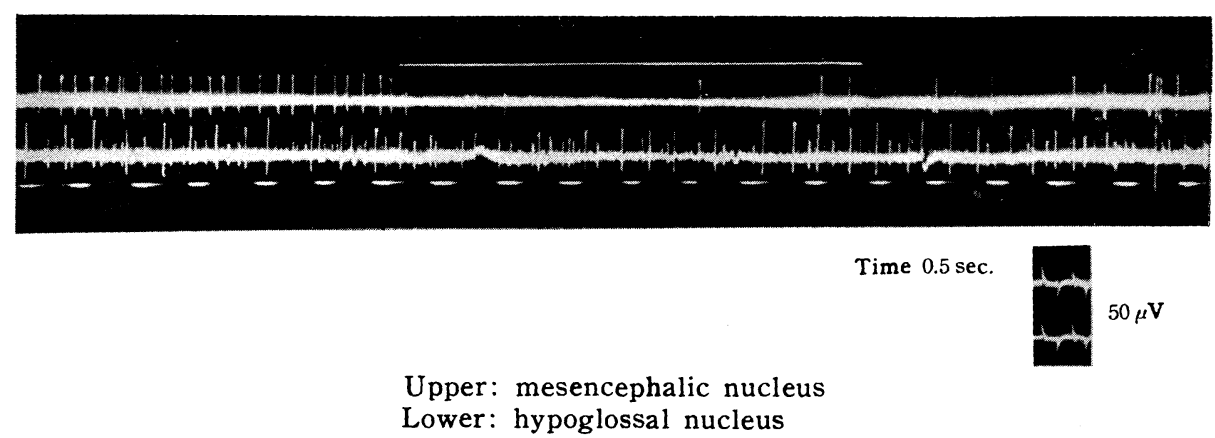

FIG. 5. Simultaneous recording of responses in midbrain and medulla by elevating the jaw.

A

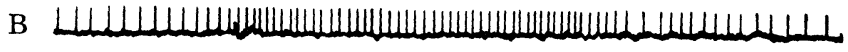

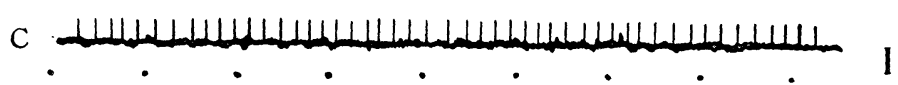

Time: 1 sec.

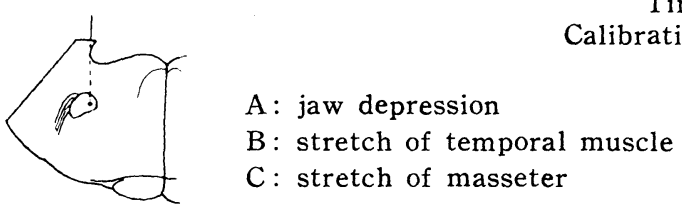

Site of the electrode tip

Fig. 6. Point responding to stretching the temporal muscle.

there were some points in the comparatively rostral part of the nucleus in which the frequency of spontaneous unit discharge was activated by the procedure of stretching only the ipsilateral temporal muscle. But in this bulbar point no response was recognized by stretching the masseter, occipito-mandibularis, limbs, by pinching the pinna or skin etc. In fig. $6, A$ is a case when the lower jaw was depressed, $B$ is a case where the temporal muscle was stretched and $C$ the masseter. The $6 \mathrm{c} / \mathrm{s}$ spontaneous unit discharge of this point was activated to about $8 \mathrm{c} / \mathrm{s}$ only in the case of stretching of the ipsilateral temporal muscle. Fig. 7 shows frequency changes of the unit discharge at this bulbar point during these procedures, and each spot in this figure is the discharge frequency value per second in process of time. As such, the response of this point aroused by depressing the lower jaw is confirmed to be the one mostly attributable to stretching of the ipsilateral temporal muscle.

In the rostral areas of the trigeminal motor nucleus there were some other points, as shown in fig. 8 , in which the discharge was activated by stretching either the ipsilateral masseter or temporal muscle respectively. But in this 


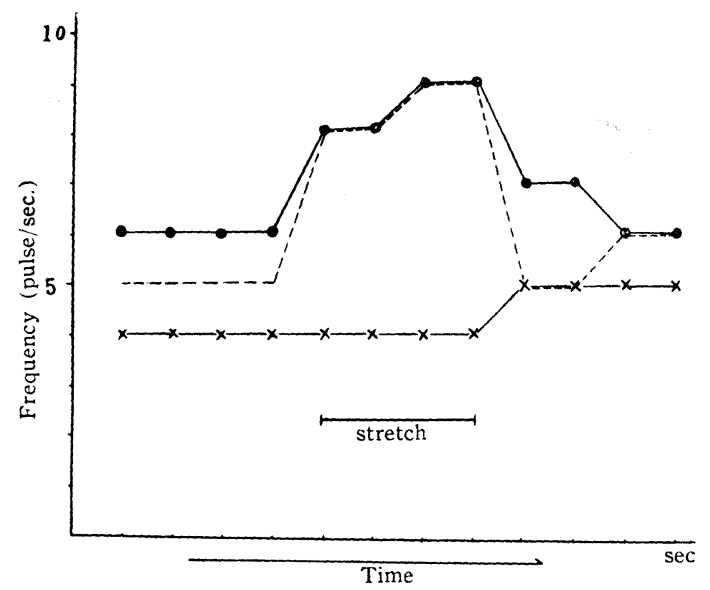

FIG. 7. Effect of jaw depression or stretching a jaw muscle on discharge frequency of some point in rostral part of the trigeminal motor nucleus.

$x$ : stretching masseter, $\bullet$ : stretching temporalis, $\cdots$ : jaw depression

\section{Sign}

Temporalis
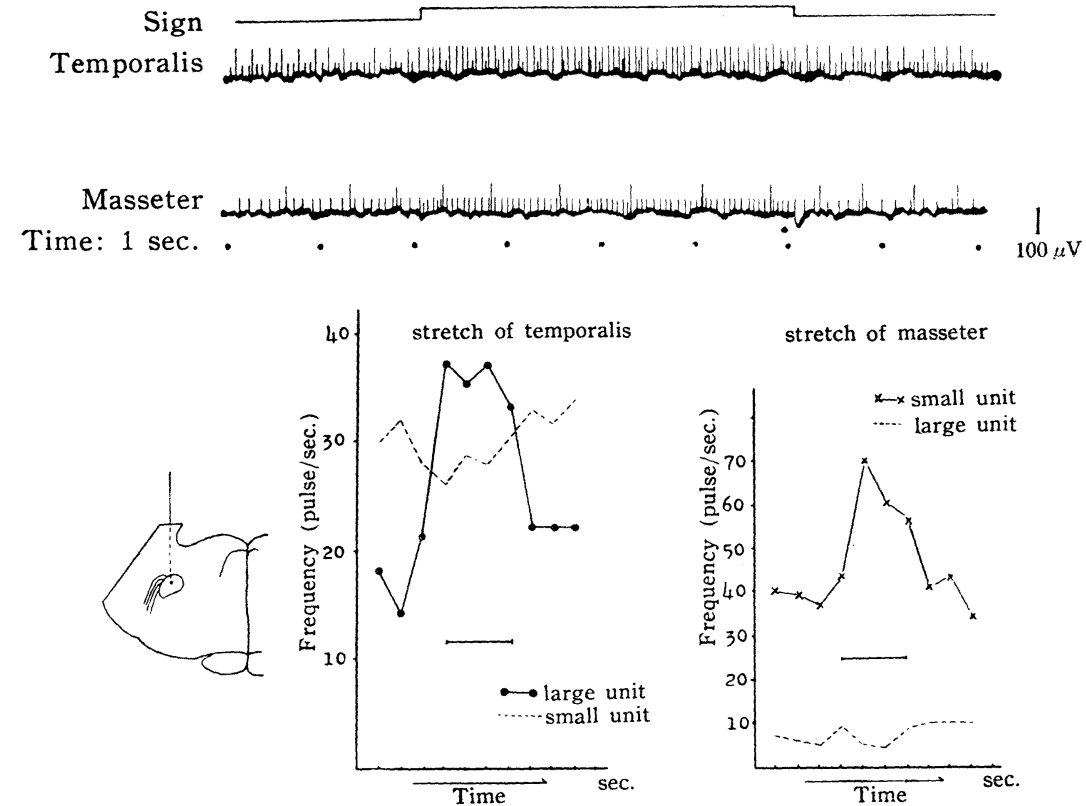

FIG. 8. The response from the rostral part of the trigeminal motor nucleus.

Upper is discharge record. Lower is the site of the recording electrode tip and interval diagrams of the two unit discharges in the case of either stretching the temporalis or masseter. 
case only an unit with $18 \mathrm{c} / \mathrm{s}$ high voltage discharge was activated to $37 \mathrm{c} / \mathrm{s}$ only by stretching the temporal muscle, and the other unit with $30 \mathrm{c} / \mathrm{s} \mathrm{low}$ voltage from this same point was not affected at all. And the latter was activated to $70 \mathrm{c} / \mathrm{s}$ only by stretching the masseter and the former unit did not respond at all to stretching of the masseter (fig. 8). The site of the recording electrode tip and the discharge frequencies of these two units are shown in a graph in lower part of this figure.

Moreover, in the comparatively caudal part of this nucleus, there were some characteristic points which showed an acceleration of discharge frequency only by elevating of the lower jaw and not by depression of the jaw. As shown in fig. 9, about the $24 \mathrm{c} / \mathrm{s}$ unit discharge of this point was activated to about $62 \mathrm{c} / \mathrm{s}$ by elevating the lower jaw. This response was not induced by either depressing the jaw or stretching the temporal muscle, however, it was induced by stretching the ipsilateral occipito-mandibular muscle which is one

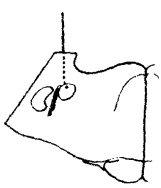

Site of the electrode tip
A

B

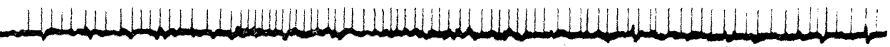

C

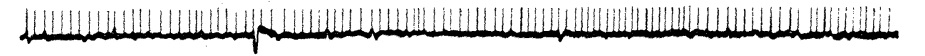

Time: $1 \mathrm{sec}$.

FIG. 9. Point responding to stretching the occipito-mandibular muscle.

A. jaw elevation

B. stretch of occipito-mandibular muscle

C. stretch of temporal muscle

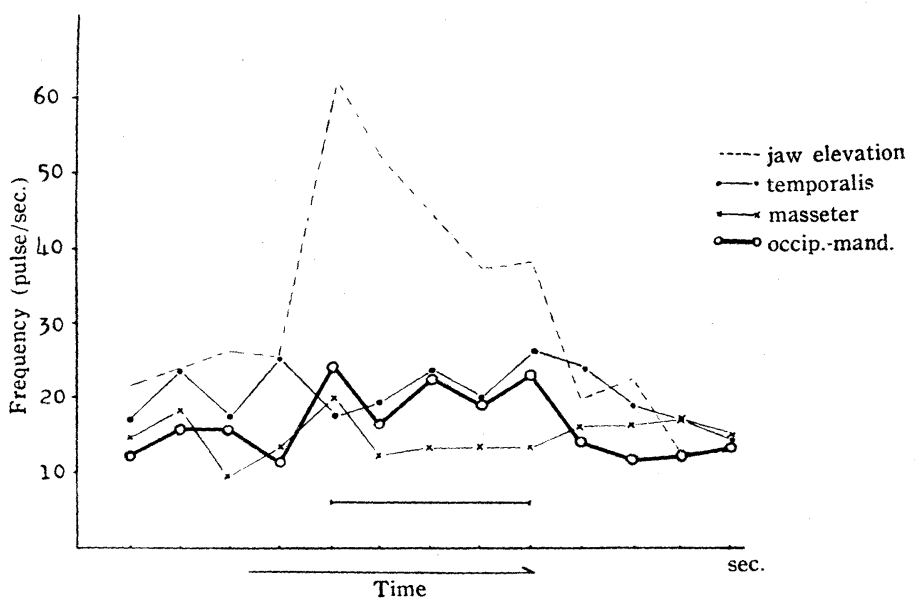

FIG. 10. Effect of jaw elevation or stretching a jaw muscle on discharge frequency of some point in caudal part of the trigeminal motor nucleus. 
of the jaw opening muscles. This relation is clearly shown in fig. 10 as the graph of discharge frequency in each case. Additionally, in the mesencephalic trigeminal nucleus, a point showing an inhibition of a spontaneous discharge by pulling of the occipito-mandibular muscle was newly found. Such points, however, are scarce in the nucleus. As such, it was confirmed that there were characteristic projections of the spindle afferent of each masticatory muscle in the trigeminal motor nucleus, and as shown in fig. 11. spots of the masseter and temporalis existed in the rather rostral part of this nucleus and the spot of the occipito-mandibularis was situated in the more caudal part of this nucleus than the former two.

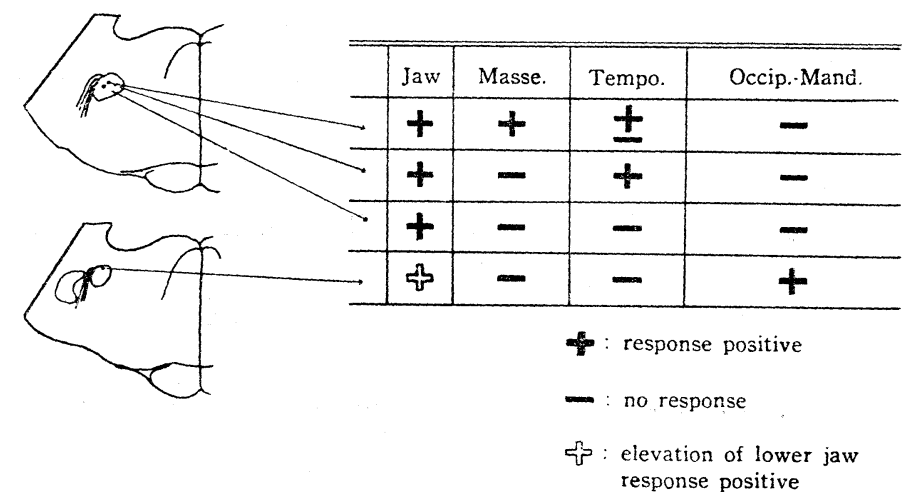

FIG. 11. Functional representation of motor nucleus of trigeminal nerve.

\section{DISCUSSION}

Three important facts were detected in this experiment. The first is the existence of very intimate functional relations between the mesencephalic nucleus and motor nucleus of the fifth cranial nerve. The second, some cells in both of these nuclei are recognized to respond to stretching of the jaw muscles, and the spindle afferent from each masticatory muscle to these cell stations has an individual representation in the nucleus. Roughly speaking, the spindle afferent from the jaw closing muscle projects to the rather rostral part of the trigeminal motor nucleus and that of the jaw opening muscle goes to the more caudal part of it. Presumably these distribution might exist also in the mesencephalic nucleus, but it was not determined in the present experiment owing to technical difficulty. The third, there was a clear reciprocal functional relationship between the mesencephalic trigeminal nucleus and the bulbar hypoglossal nucleus. These three points will be discussed here.

The main fiber masses of the mesencephalic root of the fifth nerve have been declared to enter the branches of the mandibular nerve $(3,4,5)$. And the possible functions of the spinal dorsal-root ganglia which has been demonstrated by Warrington and Griffith (1904) may well be applied to the cells of the mesencephalic nucleus, since there is a marked similarity between the 
cells of both location $(6,7,8,9,10)$. As such, the conception that the functional character of the mesencephalic trigeminal nucleus and root are a proprioceptive nerve carring the sense from the masticatory muscles are based chiefly on morphological data. Recently, this conception was clearly confirmed physiologically by Corbin and Harrison (11), Cooper, Daniel and Whitteridge (2) and Kawamura et al. (1). Further, the discharge pattern of the mesencephalic and motor nuclei of the fifth nerve by stretching the masticatory muscle recorded in the present experiment was similar to the report of Eldred, Granit and Merton (12) that has shown the activity of the spindle afferent in a dorsal root filament by stretching the limb muscle. After Cajal's report (1896) many morphologists $(4,11,13,14,15,16)$ have demonstrated that the cells of the mesencephalic trigeminal nucleus are unipolar or pseudo-unipolar neurons and the process that give reflex collaterals to the motor ganglion cells of the fifth nerve before descending into the minor portion of the trigeminal root. This morphological concept was also accepted physiologically through the present result.

Additionally, Szentágothai (17) has shown that the collaterals of the mesencephalic tract neurons can be traced to the ipsilateral motor nuclei of the infrahyoid muscles; to the caudal part of the hypoglossal nucleus. Since the infrahyoid muscles are one of the antagonists of the jaw closing muscles, and Szentágothai suggested these few collaterals can be considered as direct inhibitory fibers to the jaw closing muscles, it is very important that the spontaneous discharge of the hypoglossal nucleus was reciprocally inhibited when the discharges of the mesencephalic and motor nucleus of the fifth nerve were accelerated by stretching the jaw closing muscle.

The points in midbrain responding the stretching of the occipito-mandibular muscle were very few, and this well coincides with the morphological data $(14,17)$. Several samples of the background activity of the brainstem were presented in this paper, but the frequency of these spontaneous discharges varied in each point, and even in the same point the discharge frequency may change according to the condition of the animal.

In the present experiment, the discharge frequency of a single unit in the nucleus of the brainstem was slower than that seen in Cooper's report. This might be attributed to the differences of condition of the experimental animal, and in the present work decerebrate and decerebellate animals were used. Even in this experiment, in the case of non-decerebrate animals the unit discharge of the brainstem showed a more fast frequency and many units came into action. Therefore, higher centers might send activating impulses usually to the cells of the spindle afferent in the mesencephalic and motor nucleus of the fifth nerve.

The detail functional relations of the mesencephalic nucleus of the trigeminal nerve and hypoglossal nucleus are not yet known. But, at least, it may be speculated that the neural course between them is an inhibitory one, and consisted of a polysynaptic neural connection.

Florey and McLennan (18) have already reported that Factor I (inhibitory substance gained from brain) specially induced an excitation of electromyo- 
graphic responses from the tongue of the cat following topical application to the hypoglossal nuclei. We (19) have claimed also a similar phenomenon in which the spontaneous discharge of the hypoglossal nucleus is accelerated by topical application of Substance I (an inhibitory substance extracted with ethanol from dog brain by means of a method similar to Florey). Conversely, the spontaneous discharges of the mesencephalic and motor nuclei of the fifth cranial nerve are inhibited by topical application of this Substance I, however, they are accelerated by topical application of acetylcholine (These will be reported in the next paper). These facts suggest that some different chemical process is concerned with an excitation of these nuclei.

\section{SUMMARY}

In this experiment the functional relations of the mesencephalic and bulbar motor nucleus of the trigeminal nerve and the hypoglossal nucleus were analized electrophysiologically.

1. By jaw depression, background activities of the mesencephalic nucleus and bulbar motor nucleus of the fifth nerve were accelerated simultaneously, but conversely that of the hypoglossal nucleus was inhibited reciprocally.

2. There were characteristic projections of the spindle afferent of each masticatory muscle in the trigeminal motor nucleus, and presumably in the mesencephalic nucleus. The spots of the masseter and temporalis were in the more rather rostral part and that of the occipito-mandibularis was situated in the caudal part of the nuclei than the former two.

\section{REFERENCES}

1. KaWAmura, Y., FunAkoshi, M. AND Tsukamoto, S. Brainstem representation of jaw muscle activities of the dog. Jap. J. Physiol. 8: 292-304, 1958.

2. Cooper, S., DANiel, P. M. AND WhitTeridge, D. Nerve impulses in the brainstem of the goat.: Short latency responses obtained by stretching the extrinsic muscles. J. Physiol. 120: 417-490, 1953.

3. MAY, O. AND HoRsley, V. The mesencephalic root of the fifth nerve. Brain 33: 175-203, 1910.

4. WEINBERG, E. The mesencephalic root of the fifth cranial nerve.: A comparative anatomical study. J. Comp. Neurol. 46: 249-405, 1928.

5. CORBIN, K. B. Observations on the peripheral distribution of fibers arising in the mesencephalic nucleus of the fifth cranial nerve. J. Comp. Neurol. 73: 153-177, 1940.

6. AlLEN, W. F. Application of the Marchi method to the study of the radix mesencephalica trigemini in the guinea pig. J. Comp. Neurol. 30: 169-216, 1919.

7. ClARK, S. L. Nissl granules of primary afferent neurones. J. Comp. Neurol. 41: 423$451,1926$.

8. SCHNEIDER, A. J. The histology of the radix mesencephalica $n$. trigemini in the dog. Anat. Rec. 38: 321-339, 1928.

9. SHEININ, J. J. Typing of the cells of the mesencephalic nucleus of the trigeminal nerve in the dog, based on Nissl-granule arrangement. J. Comp. Neurol. 50: 109131, 1930. 
10. Peters, G. A. The presence of sensory nerve cells in the central root of the trigeminal nerve. J. Comp. Neurol. 62: 349-360, 1935.

11. CORBIN, K. B. AND HARRISON, F. Function of mesencephalic root of fifth cranial nerve. J. Neurophysiol. 3: 423-435, 1940.

12. Eldred, E., Granit, R. ANd Merton, P. A. Supraspinal control of the muscle spindles and its significance. J. Physiol. 122: 498-523, 1953.

13. WALlenberg, A. Neue Untersuchungen über den Hirnstamm der Taube, III.: Die cerebrale Trigeminuswurzel. Anat. Anz. 25: 526-528, 1904.

14. Thelander, H. E. The course and distribution of the radix mesencephalica trigemini in the cat. J. Comp. Neurol. 37: 207-220, 1924.

15. PEASON, A. A. The development and connections of the mesencephalic root of the trigeminal nerve in man. J. Comp. Neurol. 90: 1-46, 1949.

16. Caglieris, A. N. Osservazioni sul nucleo mesencefalico del trigemino. Rivista. Patolo. nerv. ment. LXXIII: 1-20, 1952.

17. Szentágothai, J. Anatomical considerations of monosynaptic reflex arcs. J. Neurophysiol. 11: 445-454, 1948.

18. Florey, E. AND MCLennan, H. Effects of an inhibitory factor (factor I) from brain on central synaptic transmission. J. Physiol. 130: 446-455, 1955.

19. Kawamura, Y., Funakoshi, M. And Takata, M. Some brain extracts and its effect on the electrical activity of the masticatory nucleus. in press. 